\title{
NEAR INFRARED EMISSION OF NEUTRAL CARBON FROM PHOTON-DOMINATED REGIONS
}

\author{
V. ESCALANTE ${ }^{1}$, A. STERNBERG ${ }^{2}$, and A. DALGARNO ${ }^{3}$ \\ 1 Instituto de Astronomía, UNAM, Ap. Postal 70-264, Mexico, DF 04510, Mexico \\ 2 School of Physics and Astronomy, Tel Aviv University, Ramat Aviv, Israel \\ 3 Center for Astrophysics, 60 Garden St. Cambridge, MA 02138, USA
}

\begin{abstract}
Detailed calculations are reported of the intensities of the near infrared forbidden lines of neutral carbon atoms at $\lambda 985.0 \mathrm{~nm}, 982.3 \mathrm{~nm}$ and $872.7 \mathrm{~nm}$ emitted from dense clouds subjected to intense radiation fields. The metastable levels that produce the lines are excited by radiative recombination of the $\mathrm{C}^{+}$ions produced by photoionization. Impacts of electrons with $\mathbf{C}$ atoms in the heated edge zones of the clouds contribute an insignificant part to the excitation. The lines observed in M42 and NGC 2024 can be interpreted as arising in gas with densities in excess of $10^{5} \mathrm{~cm}^{-3}$ and radiation fields with intensities between $10^{3}$ and $10^{6}$ times the average interstellar field intensity. Radiative recombination of $\mathrm{C}^{+}$ions may also be an important source of the emission lines detected in the planetary nebulae NGC 6270 and NGC 7027.
\end{abstract}

\section{Excitation of the NIR Forbidden Lines of $\mathbf{C}$}

The near infrared forbidden lines of neutral carbon are emitted in the transitions $2 p^{2}{ }^{3} P_{2}-{ }^{1} D_{2} \lambda 985.0 \mathrm{~nm}, 2 p^{2}{ }^{3} P_{1}-{ }^{1} D_{2} \lambda 982.3 \mathrm{~nm}$, and $2 p^{2}{ }^{1} D_{2}-{ }^{1} S_{0} \lambda 872.7 \mathrm{~nm}$. The ${ }^{1} D_{2}$ and ${ }^{1} S_{0}$ levels can be excited by electron impacts from the $C^{3} P$ ground term or by radiative recombination of $\mathrm{C}^{+}$followed by cascading. Non-hydrogenic quantummechanical calculations of the recombination and transition rates were carried out to predict the emission of the forbidden lines of C (Escalante and Victor, 1990).

When the lines are excited by radiative recombination, their intensity depends weakly on temperature and is proportional to the $\mathrm{C}^{+}$and electron column densities at low densities. Furthermore the line intensity ratio of $\lambda \mathbf{9 8 5 . 0}$ (or $\lambda \mathbf{9 8 2 . 3}$ ) to $\lambda \mathbf{8 7 2 . 7}$ is almost independent of temperature. This ratio, however, depends on density because of quenching of the upper levels. On the other hand, excitation by electron impact produces line intensities that depend strongly on temperature. If the temperature and $\mathrm{C} / \mathrm{C}^{+}$ratio are sufficiently high, electron impact becomes the dominant mechanism. Thus, observations of these lines gives a diagnostic of the excitation mechanism, the radiation field and density in the region.

\section{Results and Comparison with Observations}

UV radiation from hot stars creates transition regions in molecular cloud boundaries where hydrogen is mostly in atomic form due to photodissociation. In these photon-dominated regions, also known as $\mathrm{C}^{+}$regions or photodissociation regions, the chemistry is controlled 
by reactions initiated by photons, and, for high UV fields, the temperature reaches $1000 \mathrm{~K}$ and decreases to $\sim 100 \mathrm{~K}$ at the atomic/molecular boundary.

The calculations used models of plane-parallel regions with uniform proton density $n$ similar to those of Sternberg and Dalgarno (1989). Because of the low temperature of the region and the low $\mathrm{C} / \mathrm{C}^{+}$ratio throughout the region, the contribution of electron impact to the excitation of the lines is negligible. From the model calculations it is possible to derive minimum values for the density and radiation field intensity that are necessary to produce the observed intensities of the lines for different objects as shown in Table 1. These minimum values are consistent with the densities and radiation field intensities obtained from observations of far infrared fine-structure lines of $\mathrm{C}^{+}$, radio recombination lines of $\mathrm{C}$, and $\mathrm{H}_{2}$ emission in M 42, NGC 2024, and NGC 6720.

Table 1

\begin{tabular}{lccccc}
\hline Object & $I_{1}(\lambda 985.0 \mathrm{~nm})^{\mathrm{a}}$ & $I_{2}(\lambda 872.7 \mathrm{~nm})$ & $R=I_{1} / I_{2}$ & $n_{\min }\left(\mathrm{cm}^{-3}\right)$ & $\chi_{\min }^{\mathrm{b}}$ \\
\hline M42 pos. 24 & $5.1 \times 10^{-5}$ & $1.0 \times 10^{-5}$ & 5.1 & $10^{6}$ & $10^{3}$ \\
NGC 2024 & $4.5 \times 10^{-6}$ & $\ldots$ & $\ldots$ & $3 \times 10^{4}$ & $10^{2}$ \\
NGC 6270 & $1.3 \times 10^{-5}$ & $<1.2 \times 10^{-6}$ & $>10.8$ & $10^{5}$ & $2 \times 10^{2}$ \\
NGC 7027 & $1.5 \times 10^{-3}$ & $6.0 \times 10^{-4}$ & 2.5 & $>10^{7}$ & $>10^{5}$ \\
\hline
\end{tabular}

${ }^{a}$ Intensities are in units of $\mathrm{erg} \mathrm{cm}^{-2} \mathrm{~s}^{-1} \mathrm{sr}^{-1}$. References for the intensities are given by Escalante, Sternberg, and Dalgarno (1991).

${ }^{b} \chi_{\min }=1$ for the average interstellar field (Draine, 1978)

The line ratio $R=\lambda 985.0 / \lambda 872.7$ is a good diagnostic for the excitation mechanism although the $\lambda \mathbf{8 7 2 . 7}$ line intensity has more observational uncertainties. $R=4.7$ for excitation by recombination at low electron densities. The low value of $R$ in M 47 is consistent with excitation by recombination. The high $R$ value in NGC 6720 is larger than expected if the excitation is by recombination, and allows the possibility that the lines are excited by electron impact in a warm neutral gas (Jewitt et al., 1983). The low $R$ value in NGC 7027 along with the high density derived from the models suggest that the metastable levels may be quenched by electron collisions.

\section{References}

Draine, B.T., 1978, Ap. J. Suppl., 36, 595.

Escalante, V., Sternberg, A., and Dalgarno, A., 1991, Ap. J., 375, 630.

Escalante, V., and Victor, G.A.V., 1990, Ap. J. Suppl., 73, 513.

Jewitt, D.C., Kupferman, P.N., Danielson, G.E., and Maran, S.P., 1983, Ap. J., 268, 683.

Sternberg, A., and Dalgarno, A., 1989, Ap. J., , 338, 197. 